\title{
Omega 3 Fatty Acids Reduce Bone Resorption While Promoting Bone Generation in Rat Apical Periodontitis
}

\author{
Mariane Maffei Azuma, DDS, MSc, *t João Eduardo Gomes-Filbo, DDS, MSc, PbD, * \\ Edilson Ervolino, DDS, MSc, PhD, ${ }^{\ddagger}$ Camila Barbosa Pipa, DDS, * \\ Carolina de Barros Morais Cardoso, DDS, * Ana Cristina Andrada, DMSc, ${ }^{\dagger}$ \\ Toshibisa Kawai, DDS, PbD, ${ }^{\dagger \downarrow}$ and Luciano Tavares Angelo Cintra, DDS, MSc, PbD*
}

\section{Abstract}

Introduction: This study evaluated the effects of the dietary supplement omega 3 polyunsaturated fatty acids ( $\omega-3$ PUFAs) on pulp exposure-induced apical periodontitis (AP) in rats. Methods: Twenty-eight male rats were divided into groups: control untreated rats $(C)$, control rats treated with $\omega-3$ PUFAs alone (C-0), rats with pulp exposure-induced $A P$, and rats with pulp exposure-induced AP treated with $\omega$-3 PUFAs (AP-0). The $\omega$-3 PUFAs were administered orally, once a day, for 15 days before pulp exposure and, subsequently, 30 days after pulp exposure. Rats were killed 30 days after pulp exposure, and jaws were subjected to histologic and immunohistochemical analyses. Immunohistochemical analyses were performed to detect tartrate-resistant acid phosphatase-positive osteoclasts and osteocalcinpositive osteoblasts on the bone surface of periapical area. Results were statistically evaluated by using analysis of variance and Tukey honestly significant difference, and $P<.05$ was considered statistically significant. Results: The bone resorption lesion was significantly larger in the AP group compared with AP$0, \mathrm{C}$, and $\mathrm{C}-0$ groups $(P<.05)$. The level of inflammatory cell infiltration was significantly elevated, and the number of tartrate-resistant acid phosphatase-positive osteoclasts was significantly higher in the periapical lesions of the AP group compared with AP-O, C, and C-O groups $(P<.05)$. The number of osteocalcin-positive osteoblasts was significantly increased in the AP-0 group compared with the AP group $(P>.05)$. Conclusions: Supplementation with $\omega$-3 PUFAs not only suppresses bone resorption but also promotes new bone formation in the periapical area of rats with AP in conjunction with downregulation of inflammatory cell infiltration into the lesion. (J Endod 2017;43:970-976)

\section{Key Words}

Apical periodontitis, endodontic infection, omega 3 fatty acids, osteocalcin

$\triangle$ pical periodontitis (AP) disease characterized by inflammatory bone destruction in response to intracanal bacterial infection (1). Bone remodeling processes, which are mediated by specific bone cells including osteocytes, osteoblasts, and osteoclasts, appear to be dysregulated in the apical periodontitis lesion (2). Currently, mechanical removal of infected dentin of root canals, accompanied by chemical disinfection, has been the standard procedure to treat apical periodontitis to reduce the level of inflammation at the affected site. However, to date, alternative therapies able to reduce bone resorption in apical periodontitis are not available.

Omega 3 polyunsaturated fatty acids ( $\omega$-3 PUFAs), as represented by eicosapentaenoic acid (EPA) and docosahexaenoic acid (DHA), have been accepted as an adjuvant therapy in the treatment of chronic inflammatory diseases such as rheumatoid arthritis (3), cardiovascular disease (4), and diabetes (5). In addition, $\omega$-3 PUFAs and lipid mediators derived from $\omega$ - 3 PUFAs are reported to have important roles in the prevention of pathogenic bone resorption (6). These findings indicate that dietary supplement with $\omega$-3 PUFAs inhibits the activation of proinflammatory arachidonic acid cascade, downmodulates acute inflammatory response by polymorphonuclear (PMN) leukocytes, and suppresses proliferation of lymphocytes and their production of proinflammatory cytokines $(7,8)$. The production of proinflammatory factors, such as prostaglandin $\mathrm{E}_{2}$, interleukin- $1 \beta$, and tumor necrosis factor-alpha, as a consequence of arachidonic acid cascade, acute inflammatory response by PMN, and lymphocyte activation, is known to promote osteoclastogenesis while suppressing osteoblastogenesis $(9,10)$. Many studies have reported the positive effects of $\omega$-3 PUFAs as an adjunct therapy for periodontal disease (11-15). However, to the best of our knowledge, none of these studies has ever investigated the effect of $\omega$-3 PUFAs on periapical bone

From the *Department of Endodontics, Araçatuba Dental School, Universidade Estadual Paulista (UNESP), Araçatuba, São Paulo, Brazil; ${ }^{\dagger}$ Department of Immunology and Infectious Diseases, The Forsyth Institute, Cambridge, Massachusetts; ${ }^{\ddagger}$ Department of Basic Sciences, Araçatuba Dental School, UNESP Universidade Estadual Paulista, Araçatuba, São Paulo, Brazil; and §School of Dental Medicine, Harvard University, Boston, Massachusetts.

Address requests for reprints to Dr Luciano Tavares Angelo Cintra, Department of Endodontics, Araçatuba School of Dentistry, UNESP Universidade Estadual Paulista, Araçatuba, SP, Brazil. E-mail address: lucianocintra@hotmail.com

0099-2399/\$ - see front matter

Copyright (c) 2017 American Association of Endodontists.

http://dx.doi.org/10.1016/j.joen.2017.01.006 
resorption processes in either human patients with apical periodontitis or animal models of endodontic infection.

Osteocalcin $(\mathrm{OCN})$ plays a key role as a calcium-binding protein and is one of the most abundant non-collagenous proteins in bone tissue (16). The expression of OCN is considered to be a biomarker of bone formation (17). Especially, OCN is produced by osteoblast cells in their late stage of differentiation (18). Although it has been reported that $\omega$-3 PUFAs can promote homeostatic osteoblastogenesis-mediated new bone formation (19), the effects of $\omega-3$ PUFAs on the expression of OCN, as well as on pathogenic bone remodeling, remain unclear.

Therefore, this study aimed to evaluate the effect of the dietary supplement $\omega-3$ PUFAs on the pathogenic bone resorption induced in a rat model of apical periodontitis by pulp exposure-elicited endodontic infection. To evaluate the impact of $\omega-3$ PUFAs on dysregulated bone remodeling processes, tartrateresistant acid phosphatase (TRAP)-positive osteoclast cells, as well as OCN-positive osteoblasts, were monitored through histologic and immunohistochemical analyses.

\section{Experimental Animals}

\section{Materials and Methods}

The experimental protocol was approved by the Institutional Ethics Committee (CEUA 2014-00550) of UNESP-Universidade Estadual Paulista, Sao Paulo, Brazil and conducted in accordance with relevant guidelines. Twenty-eight 6-week-old male Wistar rats (Rattus norvegicus albinus) weighing 200-250 g each were used in this study. The rats were housed in a mini-isolator (Alesco, São Paulo, SP, Brazil) in temperature-controlled rooms and given ad libitum access to water and food.

The rats were randomly assigned into 4 groups (7 rats/group):

1. Control untreated rats (C)

2. Healthy rats treated with $\omega$-3 PUFAs (C-0)

3. Rats with pulp exposure-induced AP

4. Rats with pulp exposure-induced AP treated with $\omega$-3 PUFAs (AP-0)

\section{Supplementation with $\omega-3$ PUFAs}

Rats in the C-O and AP-0 groups were orally gavaged with $\omega-3$ PUFAs (Omega 3 Catarinense-Laboratório Catarinense S.A, Joinville, SC, Brazil) (water solution, $40 \mathrm{mg} / \mathrm{kg} ; 60 \% \mathrm{EPA}$ and $40 \%$ DHA, once a day), whereas rats in groups $\mathrm{C}$ and AP received control distilled water during 15 days before AP induction (prophylactic administration) and 30 days after AP induction (therapeutic administration). As a consequence, rats were treated with either $\omega-3$ PUFAs or control water for a total period of 45 days $(12,13)$.

\section{Induction of AP}

Rats were anesthetized with ketamine $(87 \mathrm{mg} / \mathrm{kg}$; Francotar; Virbac do Brazil Ind. e Com. Ltda., Roseira, SP, Brazil) and xylazine (13 mg/kg; Rompun; Bayer S. A., São Paulo, SP, Brazil). The pulp of the right mandibular first molar was exposed by using a dental round bur (Broca Ln Long Neck; Maillefer, Dentisply Ind e Com Ltda, Petrópolis, SP, Brazil) (groups AP and AP-0) for the development of AP. The coronal pulp tissue was exposed to the oral cavity for 30 days.

\section{Immunohistologic Analyses}

Mandibles sampled from killed rats were decalcified in 10\% EDTA for 30 days and submitted to conventional histologic processing for the creation of paraffin-embedded tissue sections. Semi-serial sections $(4 \mu \mathrm{m})$ were performed in the laterolateral direction, allowing sectioning of the mandibular first molar in its longitudinal axis. Sections were stained with hematoxylin-eosin (H\&E) or submitted to immunohistochemistry by using an indirect immunoperoxidase technique for TRAP (primary antibody goat anti-TRAP SC 30832; Santa Cruz Biotechnology, Santa Cruz, CA) and primary antibody rabbit anti-OCN SC-30044 for OCN, following the previously described protocol (20). Histopathologic, histometric, and immunohistochemical analyses were performed by a certified histologist (E.E.) who was blinded to the experimental groups.

Histologic analysis was conducted by using the following parameters: nature and extension of inflammation, presence and extension of necrosis, state of vasculature, and pattern of cellularity of dental and periapical tissues.

The intensity of inflammatory infiltration was graded as follows: absent ( 0 to few inflammatory cells: score 1$)$, mild ( $<25$ inflammatory cells: score 2), moderate (25-125 inflammatory cells: score 3$)$, or severe (>125 inflammatory cells: score 4 ).

For AP and AP-O groups, the area of periapical lesion associated with the distal root of the mandibular first molars was histometrically measured. The area was calculated by rounding up the lesion boundary, considering the outer external surface of alveolar bone, and it was expressed in square micrometers. For each rat, 7 serial histologic sections were measured histometrically by using an image processing system that consisted of a light microscope (DM $4000 \mathrm{~B}$; Leica), a color camera (DFC 500; Leica, Wetzlar, Germany), a color image processor (Leica Qwin V3 software; Leica), and a personal computer (Intel Core I5, Intel Corp, Santa Clara, CA; Windows 10, Microsoft Corp, Redmond, WA). The AP areas were determined for each side, and the average value (mean \pm standard deviation) was calculated for each experimental group.

The numbers of osteoclasts and osteoblasts were analyzed in the histologic section used for histometric analysis. The perimeter was calculated by contouring the boundary of the AP with the aid of Leica Microsystems software. The numbers of TRAP-positive multinucleated cells as well as OCN-positive cells were calculated in the perimeter and expressed in cells/millimeter.

\section{Statistical Analysis}

The numbers of TRAP-positive cells, OCN-positive cells, and lesion size were statistically determined by using analysis of variance for multiple comparisons, and the Tukey test was used for pairwise comparisons at 5\% significance. Histologic findings were analyzed with the Kruskal-Wallis test. The Dunn method was used for pairwise comparisons at $5 \%$ significance. Statistical analyses were performed by using SigmaPlot software (San Jose, CA).

\section{Results \\ Histopathologic and Histometric Analysis}

To evaluate the effects of $\omega$-3 PUFAs on cell infiltrate in the AP, histologic images of H\&E-stained periapical area in the different experimental groups of rats killed at day 30 were performed and are shown in Figure 1. No sign of inflammation was noted in the periapical tissue of $\mathrm{C}$ or C-O groups (Fig. $1 A$ and $B, E$ and $F$ ). However, in the AP and AP-O groups, the dental pulp showed signs of total necrosis at 30 days after pulp exposure, and the enlargement of periapical space between bone and cementum of tooth apex was observed, indicating that bone surrounding apex was pathogenically resorbed in response to pulp exposure (Fig. $1 C$ and $D, G$ and $H$ ). Furthermore, these lesions 

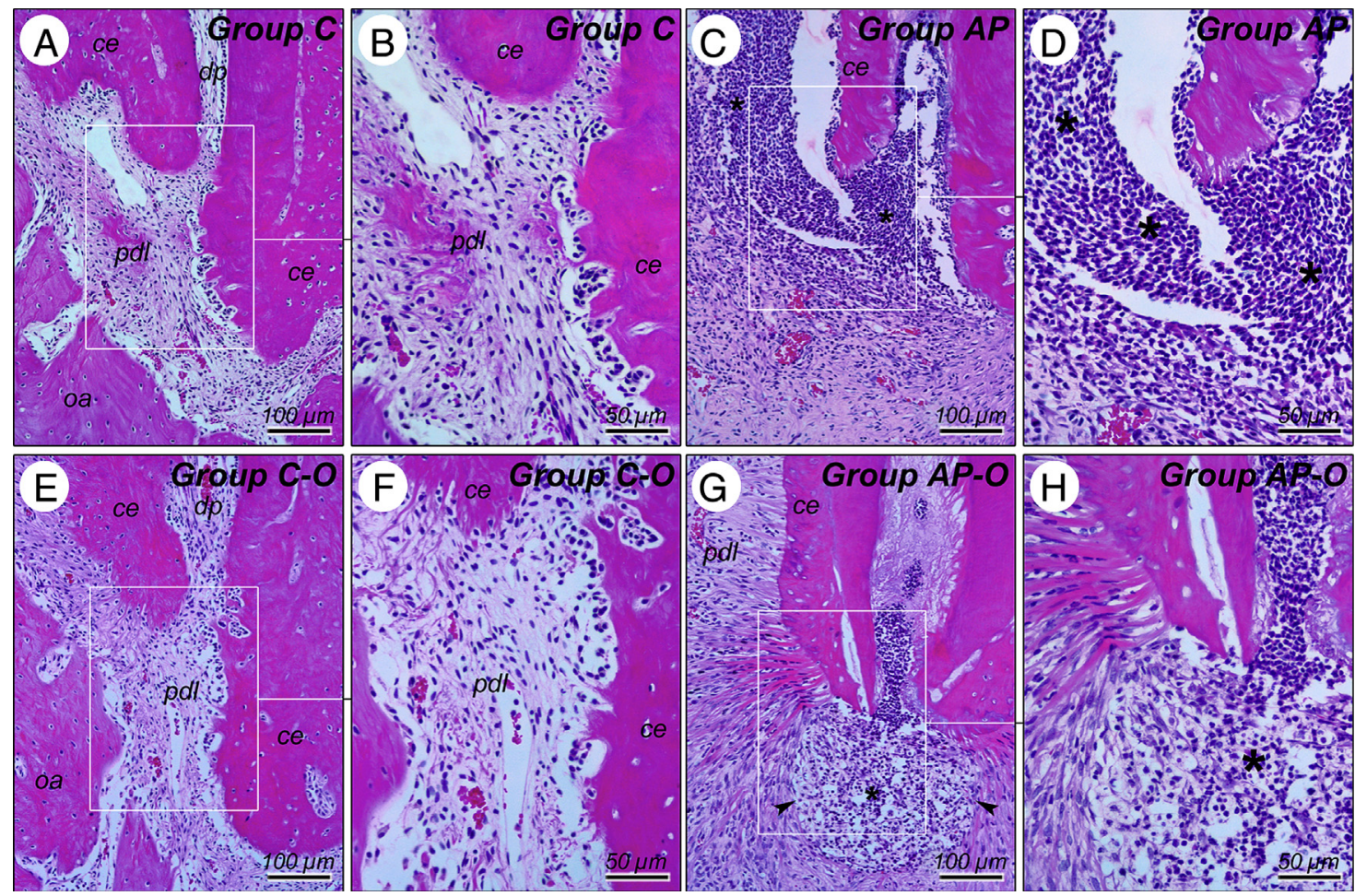

Figure 1. Histologic images of periapical lesions at 30 days after pulp exposure (H\&E staining). ( $A$ and $B$ ) Group C. Periapical region is free of inflammatory infiltrates (original magnification, $\times 100$ and $\times 200$, respectively). $(E$ and $F)$ Group C- 0 . Similar to C, the periapical region is free of inflammatory infiltrates (original magnification, $\times 100$ and $\times 200$, respectively). $(C$ and $D)$ Group AP. Intense infiltration of inflammatory cells in the area surrounding tooth apex can be observed. Severe disorganization of periodontal ligament and large bone resorption area are visible (hematoxylin; original magnification, $\times 100$ and $\times 200$, respectively). ( $G$ and $H$ ) Group AP-0. Compared with AP, a decreased level of inflammatory infiltrates is observed in periodontal ligament region. Size of bone resorption is also diminished compared with AP group (hematoxylin; original magnification, $\times 100$ and $\times 200$, respectively). Arrowheads indicate margins of apical periodontitis. dp, dental pulp; pdl, periodontal ligament; ce, cementum; oa, alveolar bone. *Site of infiltration of inflammatory cells.

were primarily composed of inflammatory infiltrates, including neutrophils and mononuclear cells. Statistical analysis showed that the magnitude of bone loss was higher in the AP group compared with the AP-0, $\mathrm{C}-0$, and $\mathrm{C}$ groups $(P<.05)$. The intensity of inflammatory infiltration was greater in the AP group (Fig. $1 C$ and $D$ ) compared with $\mathrm{C}$ (Fig. $1 A$ and $B$ ), C-O (Fig. $1 E$ and $F$ ), and AP-O groups (Fig. $1 G$ and $H)(P<.05)$ (Table 1).

To evaluate the effects of $\omega$-3 PUFAs in the bone resorption, the area of AP was measured histometrically. The area of periapical lesion of mandibular first molar was larger in the AP-O (Fig. $1 G$ and $H$ ) and AP (Fig. $1 C$ and $D$ ) groups when compared with the $\mathrm{C}$ (Fig. $1 A$ and $B$ ) and C-0 groups (Fig. $1 E$ and $F)(P<.05)$. In addition, AP was more evident in the AP group when compared with the AP-0 group $(P<.05)$ (Fig. $1 C$ and $D, G$ and $H$, Table 1 ).

\section{Histologic Evaluation of TRAP-positive Osteoclasts and OCN-positive Gells That Emerged in Periapical Lesion}

To evaluate bone remodeling and effect of $\mathrm{w}-3$ PUFAs on osteoclastogenesis, we performed TRAP staining.

The histochemistry images of TRAP staining are shown in Figure 2. The number of TRAP-positive multinucleated cells per millimeter on the alveolar bone surface of rats induced of AP was higher in the AP (Fig. $2 E$, $F$, and $I$ ) and AP-O (Fig. 2G-I) groups compared with the C (Fig. 2A, $B$, and $I$ ) or C-O groups (Fig. $2 C, D$, and $I)(P<.05)$. In addition, the AP group showed a higher number of TRAP-positive cells when compared with the AP-0 group $(P<.05)$ (Fig. $2 E-I)$.

To evaluate bone remodeling and effect of $\omega$-3 PUFAs on osteoblastogenesis, we performed histochemistry to detect OCN-positive cells. The histochemistry images of OCN-positive cells are shown in

TABLE 1. Scores and Median of Intensity of Inflammatory Cells and AP Area $\left(\mu \mathrm{m}^{2}\right)$ according to the Groups

\begin{tabular}{|c|c|c|c|c|c|}
\hline Histologic parameters & \multicolumn{4}{|c|}{ Experimental groups } & Statistical analysis \\
\hline Intensity of inflammatory infiltration & $\mathrm{C}$ & $\mathrm{C}-\mathrm{O}$ & AP & AP-O & \\
\hline 1: absent & $7 / 7$ & $7 / 7$ & $0 / 7$ & $5 / 7$ & Kruskal-Wallis \\
\hline 2: mild & $0 / 7$ & $0 / 7$ & $0 / 7$ & $2 / 7$ & $P<.05$ \\
\hline 3: moderate & $0 / 7$ & $0 / 7$ & $3 / 7$ & $0 / 7$ & \\
\hline 4: severe & $0 / 7$ & $0 / 7$ & $4 / 7$ & $0 / 7$ & \\
\hline Median* & $1^{\mathrm{a}}$ & $1^{\mathrm{a}}$ & $4^{\mathrm{b}}$ & $1^{\mathrm{a}}$ & \\
\hline AP $\left(\times 10^{4} \mu \mathrm{m}^{2} \pm\right.$ standard deviation*) & $13.20 \pm 1.67^{a}$ & $12.78 \pm 1.71^{\mathrm{a}}$ & $129.08 \pm 19.38^{b}$ & $43.94 \pm 27.90^{c}$ & $\begin{array}{l}\text { Tukey } \\
P<.05\end{array}$ \\
\hline
\end{tabular}

AP, rats with pulp exposure-induced AP; AP-0, rats with pulp exposure-induced AP treated with $\omega-3$ PUFAs; C, control untreated rats; C-0, healthy rats treated with $\omega-3$ PUFAs.

*Different superscript letters indicate significant statistical differences in rows $(P<.05)$ 

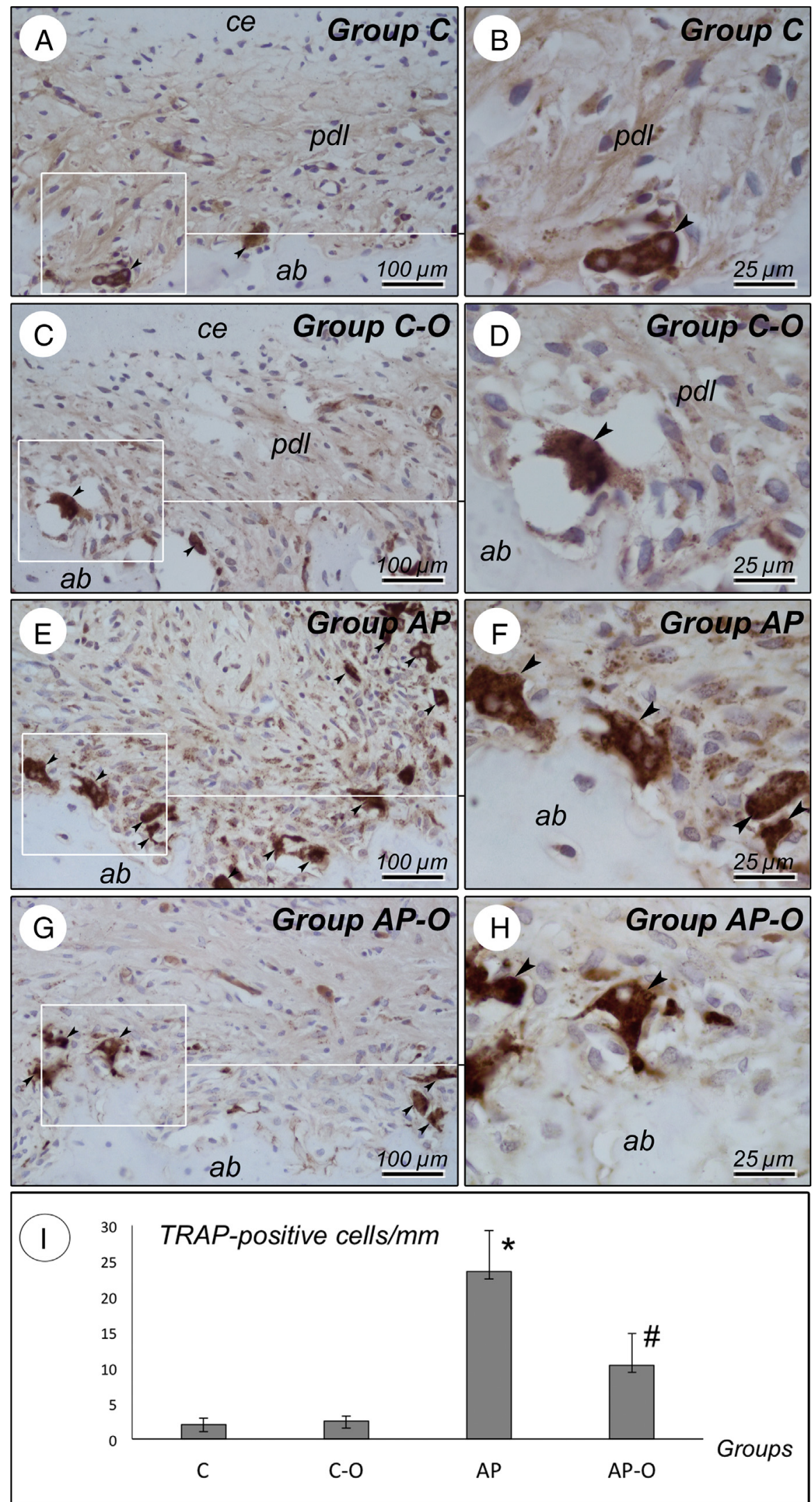

Figure 2. Histologic images and number (median and standard deviation) of TRAP-positive multinucleated cells (arrowheads) stained in the periapical bone at 30 days after pulp exposure groups. Representative images control groups $\mathrm{C}(A$ and $B)$ and C-O $(C$ and $D)$, as well as apical periodontitis groups AP $(E$ and $F)$ and AP-0 $(G$ and $H)$, are shown. ab, alveolar bone; pdl, periodontal ligament. After staining the section with TRAP, nuclei were counterstained with hematoxylin. Original magnification, $\times 100(A, C, E$, and $G)$ and $\times 400(B, D, F$, and $H)$. The numbers of TRAP-positive cells per millimeter in the perimeter of AP in the distal root of the mandibular first molar in the experimental groups are shown $(I)$. *Statistically significant difference between indicated group and C, C-0, and AP-0 groups $(P<.05)$. " Statistically significant difference between indicated group and $\mathrm{C}, \mathrm{C}-0$, and AP groups $(P<.05)$. 

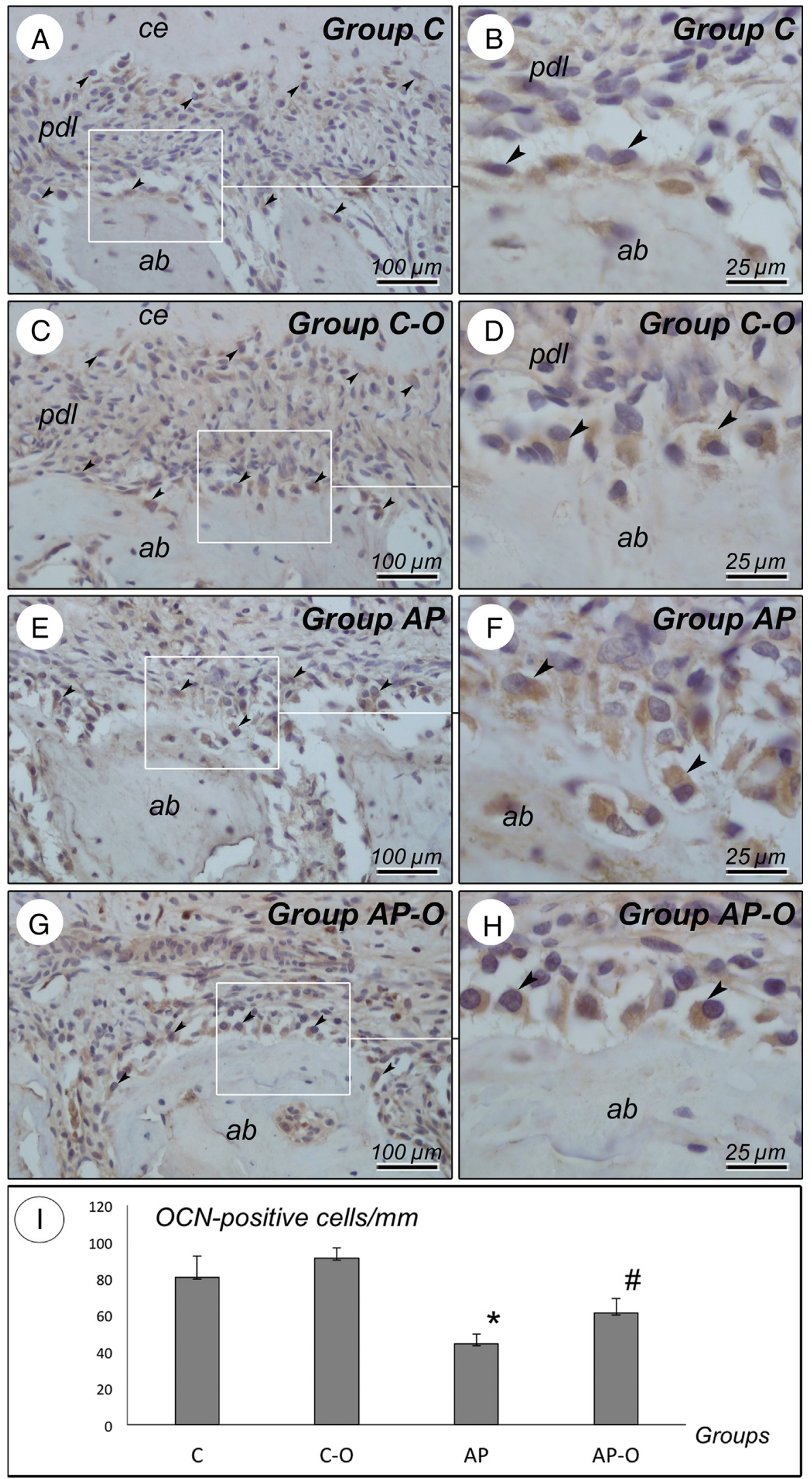

Figure 3. Histologic images and number (median and standard deviation) of OCN expressed in osteoblasts (arrowheads). Representative images of non-exposed control groups $\mathrm{C}(A$ and $B)$ and $\mathrm{C}-\mathrm{O}(C$ and $D)$, as well as apical periodontitis groups AP $(E$ and $F)$ and AP-O $(G$ and $H)$, are shown. ab, alveolar bone; pdl, periodontal ligament. After staining the section with $O C N$, nuclei were counterstained with hematoxylin. Scale bars: original magnification: $C-E, \times 100(A, C$, $E$, and $G)$ and $\times 400(B, D, F$, and $H)$. The numbers of OCN-positive cells per millimeter in the perimeter of AP in distal root of mandibular first molar in the experimental groups are shown $(I)$. *Statistically significant difference between indicated group and C, C-0, and AP-0 groups $(P<.05)$. " Statistically significant difference between indicated group and $\mathrm{C}, \mathrm{C}-0$, and AP groups $(P<.05)$. 
Figure 3. The number of OCN-positive cells per millimeter on the alveolar bone surface of rats induced of AP was higher in the C (Fig. 3A, $B$, and $I$ ) and C-O (Fig. 3C, D, and $I$ ) groups compared with the AP (Fig. 3E, $F$, and $I$ ) or AP-O groups (Fig. $3 G-I)(P<.05)$. In addition, the AP-O group showed a higher number of OCN-positive cells when compared with the AP group $(P<.05)$ (Fig. $3 E-I)$.

\section{Discussion}

In the present study, systemic administration of $\omega-3$ PUFAs not only inhibited osteoclastogenesis but also promoted osteoblastogenesis in the context of bone resorption lesion of apical periodontitis, suggesting that $\omega-3$ PUFAs can act on 2 different bone cells and ameliorate the pathogenic bone loss outcomes of this disease. The pathogenic bone resorption induced in the AP group by pulp exposure was significantly suppressed by systemic administration with $\omega$-3 PUFAs in the AP-0 group. This result agrees with previous studies performed in the rat model of periodontitis and human patients with periodontitis (14, $21,22)$. However, other studies concluded that dietary supplement with $\omega$-3 PUFAs had no effect on bone resorption in human patients with periodontitis $(12,13,23)$. Nonetheless, none of these studies has ever addressed the effects of $\omega$-3 PUFAs on pathogenic bone outcomes of apical periodontitis.

Rats that received prophylactic administration of $\omega-3$ PUFAs in addition to therapeutic administration showed better outcomes in suppressing the bone resorption induced in AP compared with rats that received therapeutic administration alone $(12,13)$. To explain, prophylactic administration of $\omega-3$ PUFAs for 2 weeks allowed the accumulation of EPA and DHA in the cell membrane (24), eventually promoting the robustness of membrane stability and fluidity in response to inflammatory challenge by the induction of periodontitis. It would be intriguing to examine in a future study whether prophylactic administration of $\omega$-3 PUFAs can also increase the efficacy of $\omega$-3 PUFAs on pathogenic bone loss induced by pulp exposure.

The present study corroborates the findings (25) in which rats induced of endodontic infection were supplemented with fish oil. Compared with experimental rats that received placebo treatment, a decreased number of osteoclasts was seen in periapical lesions of rats that received $\omega$-3 PUFAs. Some in vitro studies have also demonstrated that $\omega$-3 PUFAs could decrease osteoclastogenesis (26-29). It is well-established that the metabolites of polyunsaturated fatty acids, eg, prostaglandin, hydroxyeicosatetraenoic acids, and leukotrienes, act on osteoclast progenitors and suppress their receptor activator of nuclear factor kappa B ligand-mediated differentiation and activation $(30,31)$. According to an in vitro study, the effects of DHA on osteoclastogenesis are, at least in part, mediated by lipoxygenase, a metabolic derivative of DHA (32).

The level of OCN is considered a marker of new bone formation (19). Thus, our results showing an elevated number of OCN-positive osteoblasts in the $\omega-3$ PUFAs-treated AP rats suggested that $\omega$-3 PUFAs could also improve new bone formation, even in the context of inflammatory bone resorption lesion. Further studies to elucidate the molecular mechanisms of bone formation should be performed to verify this hypothesis.

In addition, we found a greater number of inflammatory infiltrates in the periapical area of the AP group, causing more severe inflammatory infiltration, when compared with AP-O and C groups. Inflammation is characterized by a complex sequence of events involving alterations in the inflammatory network, as well as rearrangement of innate immune cell populations and changes in their activation status (33). Therefore, the presence of inflammatory cells in the AP and AP-O groups indicates inflammatory reaction to bacterial challenge in the periapical area. Reduced inflammatory status in the AP-0 group supports some studies showing the effects of $\omega-3$ PUFAs during inflammation $(34,35)$. These studies suggested that $\omega-3$ PUFAs can competitively inhibit the production of arachidonic acid metabolites via the cyclooxygenase and lipoxygenase pathways, thus reducing proinflammatory arachidonic mediators (34) and decreasing inflammatory response (35).

The findings herein presented indicate that systemic supplementation with $\omega-3$ PUFAs can decrease bone resorption in the periapical area of rats with apical periodontitis, as well as the number of infiltrating inflammatory cells and the number of osteoclasts. Furthermore, $\omega-3$ PUFAs promoted osteoblastogenesis in the lesion. In summary, this study lays the groundwork for further investigation of $\omega-3$ PUFAs as a therapeutic regimen for apical periodontitis.

\section{Acknowledgments} 03054-0).

This study was supported by FAPESP (2013/26390-0 and 2015/ The authors deny any conflicts of interest related to this study.

\section{References}

1. Kawashima N, Okiji T, Kosaka T, et al. Kinetics of macrophages and lymphoid cells during the development of experimentally induced periapical lesions in rat molars: a quantitative immunohistochemical study. J Endod 1996;22:311-6.

2. Xiong H, Wei L, Peng B. Immunohistochemical localization of IL-17 in induced rat periapical lesions. J Endod 2009;35:216-20.

3. Lee YH, Bae SC, Song GG. Omega-3 polyunsaturated fatty acids and the treatment of rheumatoid arthritis: a meta-analysis. Arch Med Res 2012;43:356-62.

4. Jain AP, Aggarwal KK, Zhang PY. Omega-3 fatty acids and cardiovascular disease. Eur Rev Med Pharmacol Sci 2015;19:441-5.

5. Farsi PF, Djazayery A, Eshraghian MR, et al. Effects of supplementation with omega-3 on insulin sensitivity and non-esterified free fatty acid (NEFA) in type 2 diabetic patients. Arq Bras Endocrinol Metabol 2014;58:335-40.

6. Maggio M, Artoni A, Lauretani F, et al. The impact of omega-3 fatty acids on osteoporosis. Curr Pharm Des 2009;15:4157-64.

7. Alam SQ, Bergens BM, Alam BS. Arachidonic acid, prostaglandin E2 and leukotriene $\mathrm{C} 4$ levels in gingival and submandibular salivary glands of rats fed diets containing n-3 fatty acids. Lipids 1991;26:895-900.

8. Fernandes G, Venkataraman J. Role of omega-3 fatty acids in health and disease. Nutr Res 1993;13:19-45.

9. Shen CL, Peterson J, Tatum OL, et al. Effect of long-chain n-3 polyunsaturated fatty acid on inflammation mediators during osteoblastogenesis. J Med Food 2008;11: 105-10.

10. Nakanishi A, Iitsuka N, Tsukamoto I. Fish oil suppresses bone resorption by inhibiting osteoclastogenesis through decreased expression of M-CSF, PU.1, MITF and RANK in ovariectomized rats. Mol Med Rep 2013;7:1896-903.

11. Vardar S, Buduneli E, Türkoğlu 0 , et al. Therapeutic versus prophylactic plus therapeutic administration of omega3 fatty acid on endotoxin-induced periodontitis in rats. J Periodontol 2004;75:1640-6.

12. Vardar-Sengül S, Buduneli N, Buduneli E, et al. Dietary supplementation of omega-3 fatty acid and circulating levels of interleukin-1beta, osteocalcin, and C-reactive protein in rats. J Periodontol 2006;77:814-20.

13. Kesavalu L, Vasudevan B, Raghu B, et al. Omega-3 fatty acid effect on alveolar bone loss in rats. J Dent Res 2006;85:648-52.

14. Iwasaki M, Yoshihara A, Moynihan P, et al. Longitudinal relationship between dietary $\omega 3$ fatty acids and periodontal disease. Nutrition 2010;26:1105-9.

15. Deore GD, Gurav AN, Patil R, et al. Omega 3 fatty acids as a host modulator in chronic periodontitis patients: a randomised, double-blind, placebo-controlled, clinical trial. J Periodontal Implant Sci 2014;44:25-32.

16. Lian JB, Gundberg CM. Osteocalcin: biochemical considerations and clinical applications. Clin Orthop Relat Res 1988;226:267-91.

17. Shibutani T, Inuduka A, Horiki I, et al. Bisphosphonate inhibits alveolar bone resorption in experimentally-induced peri-implantitis in dogs. Clin Oral Implants Res 2001;12:109-14.

18. Zoch ML, Clemens TL, Riddle RC. New insights into the biology of osteocalcin. Bone 2016;82:42-9.

19. Fong L, Muhlhausler BS, Gibson RA, et al. Perinatal maternal dietary supplementation of $\omega 3$-fatty acids transiently affects bone marrow microenvironment, osteoblast 
and osteoclast formation, and bone mass in male offspring. Endocrinology 2012; $153: 2455-65$.

20. Garcia VG, Longo M, Gualberto Junior EC, et al. Effect of the concentration of phenothiazine photosensitizers in antimicrobial photodynamic therapy on bone loss and the immune inflammatory response of induced periodontitis in rats. J Periodontal Res 2013;49:584-94.

21. Elkhouli AM. The efficacy of host response modulation therapy (omega-3 plus lowdose aspirin) as an adjunctive treatment of chronic periodontitis (clinical and biochemical study). J Periodontal Res 2011;46:261-8.

22. Bendyk A, Marino V, Zilm PS, et al. Effect of dietary omega-3 polyunsaturated fatty acids on experimental periodontitis in the mouse. J Periodontal Res 2009;44: 211-6.

23. Martinez GL, Koury JC, Brito F, et al. The impact of non-surgical periodontal treatment on serum levels of long chain-polyunsaturated fatty acids: a pilot randomized clinical trial. J Periodontal Res 2014;49:268-74.

24. Croft KD, Beilin LJ, Loece FM, et al. Effects of diet enriched eicosapentaenoic or docosahexaenoic acid or prostanoid metabolism in the rat. Lipids 1987;22: $647-50$.

25. Indahyani DE, Pudyani PS, Santoso AL, et al. The effect of fish oil on bone resorption following pulp exposure in rats. Dent Traumatol 2002;18:206-11.

26. Sakaguchi K, Morita I, Murota S. Eicosapentaenoic acid inhibits bone loss due to ovariectomy in rats. Prostaglandins Leukot Essent Fatty Acids 1994;50:81-4.
27. Kruger MC, Coetzer H, de Winter R, et al. Calcium, gamma-linolenic acid and eicosapentaenoic acid supplementation in senile osteoporosis. Aging (Milano) 1998;10:385-94.

28. Fernandes G, Lawrence R, Sun D. Protective role of $n-3$ lipids and soy protein in osteoporosis. Prostaglandins Leukot Essent Fatty Acids 2003;68:361-72.

29. Sun L, Tamaki H, Ishimaru T, et al. Inhibition of osteoporosis due to restricted food intake by the fish oils DHA and EPA and perilla oil in the rat. Biosci Biotechnol Biochem 2004;68:2613-5.

30. Yasuda H, Shima N, Nakagawa N, et al. A novel molecular mechanism modulating osteoclast differentiation and function. Bone 1999;25:109-13.

31. Bezerra MC, Carvalho JF, Prokopowitsch AS, et al. RANK, RANKL and osteoprotegerin in arthritic bone loss. Braz J Med Biol Res 2005;38:161-70.

32. Yuan J, Akiyama M, Nakahama K, et al. The effects of polyunsaturated fatty acids and their metabolites on osteoclastogenesis in vitro. Prostaglandins Other Lipid Mediat 2010;92:85-90.

33. Yang KK, Dorner BG, Merkel U, et al. Neutrophil influx in response to a peritoneal infection with Salmonella is delayed in lipopolysaccharide-binding protein or CD14deficient mice. J Immunol 2002;169:4475-80.

34. Calder PC. Polyunsaturated fatty acids and inflammation. Prostaglandins Leukot Essent Fatty Acids 2006;75:197-202.

35. Mukaro VR, Costabile M, Murphy KJ, et al. Leukocyte numbers and function in subjects eating n-3 enriched foods: selective depression of natural killer cell levels. Arthritis Res Ther 2008;10:R57. 